\title{
Modelling of an Eclipsing RS CVn Binary: V405 And
}

\author{
Krisztián Vida, Katalin Oláh, and Zsolt Kővári \\ Konkoly Observatory, \\ Konkoly Thege út 15-17., H-1121 Budapest, Hungary \\ email: vida, olah, kovari@konkoly.hu
}

\begin{abstract}
V405 And is an ultrafast-rotating ( $P_{\text {rot }} \approx 0.46$ days $)$ eclipsing binary. The system consists of a primary star with radiative core and convective envelope, and a fully convective secondary. Theories have shown that stellar structure can depend on magnetic activity, i.e., magnetically active M-dwarfs should have larger radii. Earlier light curve modelling of V405 And indeed showed this behaviour: we found that the radius of the primary is significantly larger than the theoretically predicted value for inactive main sequence stars (the discrepancy is the largest of all known objects), while the secondary fits well to the mass-radius relation. By modelling our recently obtained light curves, which show significant changes of the spotted surface of the primary, we can find further proof for this phenomenon.
\end{abstract}

Keywords. stars:activity, binaries: eclipsing, stars: fundamental parameters, stars: late-type, stars: spots

\section{Introduction}

V405 And is an X-ray emitting active binary detected by the ROSAT satellite (Voges et al. 1996). The first detailed study of the system was done by Chevalier \& Ilovaisky (1997), who detected an orbital period of $P_{\text {orb }}=0.465$ days, and a small, near grazing eclipse. The authors found that the primary and the secondary have spectral types of M0V and M5V, and both of them are active, as both show $\mathrm{H} \alpha$ emission. Vida et al. (2009) presented photometric $B V(R I)_{C}$ data, analysed optical spectroscopic measurements, and found, that the light curve modulation is caused by the combined effect of spottedness and binarity. Using an iterative modelling method to separately describe these two effects, the authors determined the physical properties of this binary system. The primary and the secondary component was found to have masses of $0.49 M_{\odot}$ and $0.21 M_{\odot}$ respectively, thus the primary is supposed to consist of a radiative core and a convective envelope, while the secondary is probably fully convective. The radii of the two components are $0.78 R_{\odot}$ and $0.24 R_{\odot}$. Plotting these values on the theoretical mass-radius diagram of Baraffe et al. (1998) together with other binaries, we find that the secondary fits well to this relation, but the primary has a significantly larger radius than the theoretically predicted value.

\section{Observations and analysis}

We have obtained new $B V(R I)_{C}$ photometry with the $1 \mathrm{~m}$ RCC telescope at Piszkéstető between JDs 2455148 and 2455531 (2009 November-2010 November, about 700 days after the light curves modelled in Vida et al. 2009). Previously we found the light curve to be stable (Vida et al. 2009), but during the time of the new observations the surface seemed to evolve significantly. The main spotted area moved from phase $\approx 0.5$ 

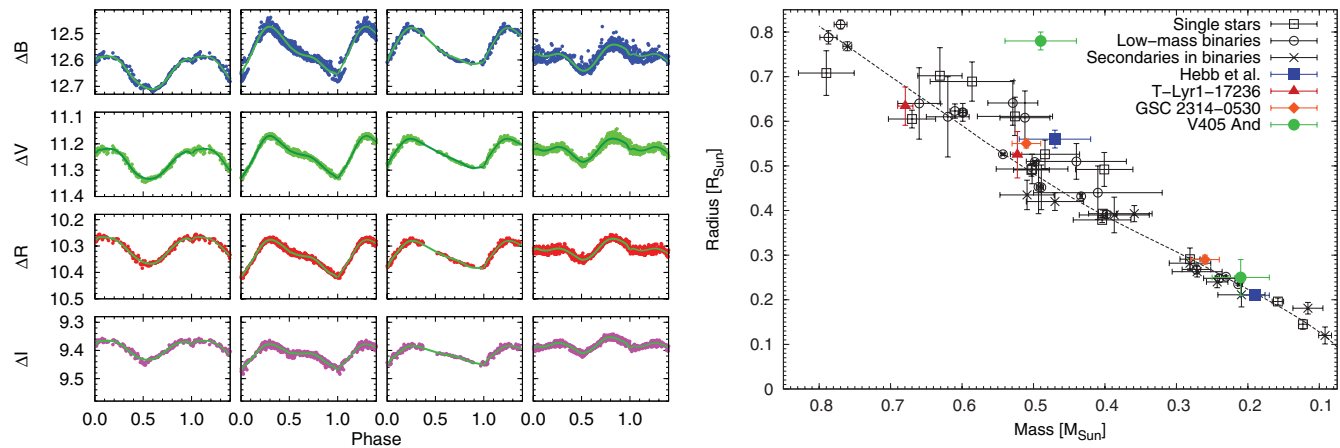

Figure 1. Left: Fits to $B V(R I)_{C}$ light curves of V405 And. The first column shows the results from Vida et al. (2009), the rest show new results. Right: Mass-radius diagram for 5 Gyr stars from Baraffe et al. (1998) (with continuous line). Dots show measurements of López-Morales (2007), Blake et al. (2008) and López-Morales et al. (2006). Filled symbols denote V405 And, GSC 2314-0530 (Dimitrov \& Kjurkchieva 2010), T-Lyr-17236 (Devor et al. 2008), and 2MASS 04463285+1901432, a binary in NGC 1647 (Hebb et al. 2006).

to phase $\approx 1$ in the first two new seasons, and in the last season two active nests were observed: around phases 0.1 and 0.5 .

Using the same modelling method described in Vida et al. (2009), we modelled the new observations using PHOEBE (Prša \& Zwitter 2005) and SpotModeL (Ribárik et al. 2003). The light curves and the fits are plotted in Fig. 1. The models fitted to the new observations left the system parameters unchanged. This indicates, that the radius of the primary is indeed much larger than expected. The two known similar binaries with similar structure, GSC 2314-0530 (Dimitrov \& Kjurkchieva 2010) and the one from Hebb et al. (2006) does not show this behaviour, although the primary of 2MASS $04463285+1901432$ (Hebb et al. 2006) has somewhat larger radius. This indicates that V405 And is a currently unique system, definitely worthy of further studies.

\section{Acknowledgements}

The authors acknowledge support from the Hungarian research grant OTKA K81421 and the "Lendület" Program of the Hungarian Academy of Sciences.

\section{References}

Baraffe, I., Chabrier, G., Allard, F., \& Hauschildt, P. H. 1998, A\& A, 337, 403

Blake, C. H., Torres, G., Bloom, J. S., \& Gaudi, B. S. 2008, ApJ, 684, 635

Chevalier, C. \& Ilovaisky, S. A. 1997, A\&A, 326, 228

Devor, J., et al., 2008, ApJ, 687, 1253

Dimitrov, D. P. \& Kjurkchieva, D. P. 2010, MNRAS, 406, 2559

Hebb, L., Wyse, R. F. G., Gilmore, G., \& Holtzman, J. 2006, AJ, 131, 555

López-Morales, M. 2007, ApJ, 660, 732

López-Morales, M., Orosz, J. A., Shaw J. S., Havelka L., Arevalo, M. J., McIntyre T., \& Lazaro, C. 2006, ArXiv Astrophysics e-prints, 0610225

Mullan, D. J. \& MacDonald, J. 2001, ApJ, 559, 353

Prša, A. \& Zwitter, T. 2005, ApJ, 628, 426

Ribárik, G., Oláh, K., \& Strassmeier, K. G. 2003, AN, 324, 202

Vida, K., Oláh, K., Kővári, Zs., Korhonen, H., Bartus, J., Hurta, Zs., \& Posztobányi, K. 2009, $A \mathscr{E} A, 504,1021$

Voges, W., Gruber, R., Haberl, F., Kuerster, M., Pietsch, W., \& Zimmermann, U. 1996, VizieR Online Data Catalog, 9011, 0 